\title{
Implicações da alteração do frênulo lingual no aleitamento materno exclusivo
}

\author{
Implications of the alteration of the lingual frenulum in exclusive breastfeeding
}

Implicaciones de la alteración del frenillo lingual en la lactancia materna exclusiva

Amanda Francesquet Melchior ${ }^{1}$, Francine Pimentel Hoher da Silveira ${ }^{1}$, Geovana de Paula Bolzan ${ }^{1}$, Berenice de Oliveira Cruz Rodrigues ${ }^{1}$, Amanda Suélen Monteiro ${ }^{1 *}$, Francielle Morais de Paula ${ }^{1}$, Giulliane Ramos Londero', Tamiris Leal Tonetto', Silvana Bastos Cogo', Cíntia Vanuza Monteiro Bugs $^{2,3}$, Graciela Dutra Sehnem¹.

\section{RESUMO}

Objetivo: Identificar as prováveis implicações da alteração de frênulo lingual no aleitamento materno exclusivo. Métodos: Trata-se de uma pesquisa qualitativa, do tipo exploratória e descritiva, realizada em um hospital público do Rio Grande do Sul, Brasil. A coleta de dados ocorreu no segundo semestre de 2020 por meio de entrevista semiestruturada via contato telefônico. Os dados foram submetidos à análise temática indutiva. Resultados: O estudo teve participação de dez mães com filhos diagnosticados com alteração de frênulo lingual, sendo que seis bebês apresentaram escore 7 no protocolo de Triagem de Avaliação do Frênulo Lingual, e três apresentaram escore 5/6. Sobre o acompanhamento pré-natal desde o primeiro trimestre de gestação, nove mães informaram ter realizado. Intercorrências como hipertensão arterial sistêmica, préeclâmpsia, infecção urinária, diabetes gestacional e crescimento intrauterino restrito foram relatadas. $\mathrm{Na}$ análise dos dados, emergiram dois temas: Implicações da alteração de Frênulo Lingual no Aleitamento Materno Exclusivo e Orientações para reavaliação do Frênulo Lingual. Conclusão: Evidenciou-se que dos 10 bebês que apresentaram anquiloglossia, sete não necessitaram realizar a frenotomia quando reavaliados no retorno da consulta médica, mas foi observado dificuldades durante a amamentação, que podem estar relacionadas à interferência do frênulo lingual durante a sucção do bebê.

Palavras-chave: Aleitamento materno, Freio lingual, Desmame.

\section{ABSTRACT}

Objective: To identify the likely implications of lingual frenulum alteration in exclusive breastf eeding. Methods: This is a qualitative, exploratory and descriptive research, carried out in a public hospital in Rio Grande do Sul, Brazil. Data collection took place in the second half of 2020 through semi-structured interviews via telephone contact. Data were subjected to inductive thematic analysis. Results: The study involved ten mothers with children diagnosed with lingual frenulum alteration, and six babies had a score of 7 in the Lingual Frenulum Assessment Screening protocol, and three had a score of 5/6. About prenatal care from the first trimester of pregnancy, nine mothers reported having performed. Intercurrences such as systemic arterial hypertension, pre-eclampsia, urinary tract infection, gestational diabetes and restricted intrauterine growth have been reported. In the data analysis, two themes emerged: Implications of the alteration of the lingual frenulum in exclusive breastfeeding and guidelines for re-evaluation of the lingual frenulum. Conclusion: Of the 10 babies who presented ankyloglossia, seven did not need to undergo frenotomy when reassessed when returning from

\footnotetext{
1 Universidade Federal de Santa Maria (UFSM), Santa Maria - RS.

*E-mail: amandasuelenmonteiro@hotmail.com

2 Universidade de Santa Cruz do Sul (UNISC), Santa Cruz do Sul - RS.

${ }^{3}$ Secretaria Municipal de Saúde, Sobradinho - RS.
}

SUBMETIDO EM: 11/2021 
the medical appointment, but difficulties during breastfeeding were observed, which may be related to interference of the lingual frenulum during the baby's suckling.

Key words: Breast feeding, Lingual frenum, Weaning.

\section{RESUMEN}

Objetivo: Identificar las posibles implicaciones de la alteración del frenillo lingual en la lactancia materna exclusiva. Métodos: Se trata de una investigación cualitativa, exploratoria y descriptiva, realizada en un hospital público de Rio Grande do Sul, Brasil. La recolección de datos se realizó en el segundo semestre de 2020 a través de entrevistas semiestructuradas vía contacto telefónico. Los datos se sometieron a análisis temático inductivo. Resultados: El estudio involucró a diez madres con niños diagnosticados con alteración del frenillo lingual, y seis bebés obtuvieron una puntuación de 7 en el protocolo de evaluación de evaluación del frenillo lingual y tres tuvieron una puntuación de 5/6. Sobre la atención prenatal desde el primer trimestre de embarazo, nueve madres informaron haberla realizado. Se han informado intercurrencias como hipertensión arterial sistémica, preeclampsia, infección del tracto urinario, diabetes gestacional y crecimiento intrauterino restringido. En el análisis de los datos surgieron dos temas: Implicaciones de la alteración del frenillo lingual en la lactancia materna exclusiva y pautas para la reevaluación del frenillo lingual. Conclusión: de los 10 bebés que presentaron anquiloglosia, siete no necesitaron ser sometidos a frenotomía al ser reevaluados al regresar de la cita médica, pero se observaron dificultades durante la lactancia, lo que puede estar relacionado con la interferencia del frenillo lingual durante la succión del bebé.

Palabras clave: Lactancia materna, Frenillo lingual, Destete.

\section{INTRODUÇÃO}

O aleitamento materno é considerado como padrão-ouro para alimentação de recém-nascidos, tendo em vista que reduz os índices de mortalidade infantil, atua na prevenção de episódios de diarreia e de infecções respiratórias, diminui o risco de colesterol alto, diabetes e Hipertensão Arterial Sistêmica (HAS), além de câncer de mama na mulher, dentre outros (MINISTÉRIO DA SAÚDE, 2015a).

Para que o aleitamento se estabeleça é necessário que o recém-nascido consiga realizar uma sucção ef etiva, aspecto este que se torna prejudicado quando a criança apresenta anquiloglossia (POMINI MC, et al., 2018; MARTINELLI, RLC et al., 2016).

A anquiloglossia, avaliada no teste da linguinha, origina-se no período embrionário em que o tecido não sofre apoptose, mantendo-se na linha média e na base da língua, caracterizando um freio curto, que ao nascimento dificulta a movimentação da língua (POMINI MC, et al., 2018).

A língua auxilia na sucção, deglutição, fala e mastigação. Ao nascimento, o bebê necessita da coordenação dos reflexos orais, vedamento labial e adequada movimentação de língua para extração do leite materno (MARTINELLI RLC, et al., 2013).

A língua, por sua vez, curva-se para cima (canolamento) e realiza movimentos anteroposteriores, associado à pega correta e movimentação adequada de mandíbula que gera a ordenha do leite. Quando ocorre a restrição da língua por alteração do frênulo, estes movimentos tornam-se limitados, podendo causar dificuldades no aleitamento materno exclusivo, fissuras mamilares, baixo ganho de peso e o risco para desmame precoce (MARTINELLI RLC, et al., 2013; ARAUJO MCM, et al., 2020).

Para a avaliação do frênulo lingual, pode-se utilizar o protocolo de Triagem Neonatal - Teste da Linguinha, que é realizado nas primeiras $48 \mathrm{~h}$ de vida do recém-nascido, ainda na maternidade, e apresenta uma avaliação em escores, sendo eles: 0 a 4, normal; 5 a 6, duvidoso; 7 ou mais, alterado com necessidade de liberação do frênulo lingual. Assim, é possível identificar os casos mais graves e indicar a frenotomia o mais precoce possível. Nos casos duvidosos, com escore de 5 ou 6 , ou quando não é possível visualizar o frênulo, 
realiza-se uma reavaliação com 30 dias de vida, orientando os pais sobre as dificuldades que podem surgir durante esse período, a fim de evitar o desmame precoce (MARTINELLI RLC, et al., 2016).

Ao considerar a integralidade de atenção à saúde da criança, as condutas voltadas para a identificação de frênulo lingual alterado podem ser realizadas por diferentes profissionais de saúde, quais sejam odontólogos, médicos pediatras, fonoaudiólogos e enfermeiros (MARTINELLI RLC, et al., 2013). A partir disso, possibilitase o fornecimento de orientações interdisciplinares desde o diagnóstico até o tratamento e cuidados pósprocedimento, quando se faz necessário realizar a freno tomia (NOGUEIRA JS, et al., 2021).

No que se relaciona ao tema do estudo, realizou-se uma busca na literatura nacional e internacional, a qual identificou que as produções disponíveis tratavam, especialmente, de questões voltadas para interferências na amamentação advindas das alterações no frênulo lingual, abrangendo desde a dor mamilar sentida pela mãe até a dor infantil ocasionada pelo intenso processo de sucção (O'SHEA JE, et al., 2017; ARAUJO MCM, et al., 2020).

Ainda, foi observado que não há um consenso na literatura sobre a anquiloglossia ser um fator predisponente ao desmame precoce e à inviabilidade do aleitamento materno exclusivo, sendo necessário que na triagem neonatal sejam analisadas as dificuldades enfrentadas durante a amamentação para determinar se a causa corresponde ou não à presença de anquiloglossia (FRAGA MRBA, et al., 2020; FUJINAGA IC, et al., 2017).

Diante do exposto, este estudo foi norteado pela seguinte questão de pesquisa: Quais as implicações da alteração de frênulo lingual no aleitamento materno exclusivo? Para respondê-la, objetivou-se identificar as possíveis implicações da alteração de frênulo lingual na continuidade do aleitamento materno exclusivo.

\section{MÉTODOS}

Trata-se de um estudo qualitativo, do tipo exploratório e descritivo, realizado em um hospital público da região central do Estado do Rio Grande do Sul, Brasil, durante o segundo semestre de 2020. A localização e captação dos participantes foram realizadas no Serviço de Arquivo Médico (SAME) e por meio de prontuários do serviço.

Para seleção das participantes, foram estabelecidos os seguintes critérios de inclusão: mães de crianças que receberam o diagnóstico de alteração de frênulo lingual, com resultado de escore 5 ou superior, e que realizavam o acompanhamento no projeto de extensão da área da Fonoaudiologia intitulado por "Habilidades orais de recém-nascidos: prevenção e promoção da saúde em motricidade orofacial na infância". Para estabelecer o escore da avaliação do frênulo lingual, foi utilizado o protocolo de Triagem "Teste da Linguinha" (MARTINELLI RLC, et al., 2016). Foram excluídas as mães de crianças que não realizaram a triagem durante a internação hospitalar ou que apresentaram normalidade no protocolo de avaliação, além de mães de crianças com diagnóstico de más-formações craniofaciais e comprometimento neurológico.

As participantes foram 10 mães de crianças diagnosticadas com alteração de frênulo lingual e a seleção ocorreu por meio da amostragem intencional (CRESWELL JW e CLARK VLP, 2013). Não houve recusas. Como forma de preservar o anonimato, as participantes foram identificadas com a utilização do sistema alf anumérico por meio do codinome "P", relativo à Participante, seguido por uma numeração arábica conforme a ordem das entrevistas.

Para a coleta de dados, empregou-se a técnica de entrevista semiestruturada, realizada via contato telefônico, com as mães de crianças diagnosticadas com alteração de frênulo lingual, após conhecimento do Termo de Consentimento Livre e Esclarecido (TCLE). Obteve-se consentimento verbal antes de iniciar a pesquisa e foi gravado em um gravador digital para fins de comprovação. Assim, de forma a respeitar os preceitos éticos, anteriormente ao início da coleta de dados via telefone, f ez-se necessário a identificação do entrevistador e o consentimento verbal de participação (PADOIN SMM, et al., 2013).

A entrevista mediada pelo telef one foi escolhida como forma de coleta de dados pois combina baixo custo, facilidade no acesso aos entrevistados e na aplicação das questões com taxas altas de respostas, confome 
interesse e disponibilidade de horário (PADOIN SMM, et al., 2013). Além disso, no contexto atual, justifica-se a realização da entrevista telefônica devido à situação sanitária imposta pela pandemia da doença causada pelo novo coronavírus (COVID-19) que possibilita manter o adequado distanciamento social. Ademais, a Instituição, cenário do estudo, suspendeu as pesquisas de campo no formato presencial durante a pandemia da COVID-19.

Para a análise dos dados, optou-se pela Análise Temática na forma indutiva, a qual corresponde ao não estabelecimento prévio de uma grade pronta de categorias ou temas para analisar os dados e é constituída por seis etapas: 1) familiarização com os dados; 2) geração de códigos; 3) busca de temas; 4) revisão dos temas; 5) definição e nomeação dos temas; 6) produção do relatório. Destaca-se que o processo de análise não precisa ser linear, ou seja, o pesquisador pode retornar a qualquer fase da análise em qualquer momento do processo, adequando-o à realidade do cenário (BRAUN V e CLARKE V, 2006).

Ressalta-se que esta pesquisa esteve em conformidade com a Resolução ํㅡ 466/12 do Conselho Nacional de Saúde, com os preceitos éticos dispostos na Resolução ㄲo 510 de 07 de abril de 2016 voltados às pesquisas nas ciências humanas e sociais e de outras que utilizam metodologias próprias dessas áreas, e seguiu as recomendações da Resolução ํㅡ 580, de 22 de março de 2018, que dispõe sobre normas e diretrizes para a execução de pesquisa em instituições que compõem o SUS (MINISTÉRIO DA SAÚDE, 2012; MINISTÉRIO DA SAÚDE, 2016; MINISTÉRIO DA SAÚDE, 2018). Obteve parecer favorável do Comitê de Ética em Pesquisa CAAE sob ํㅜ 36765920.7.0000.5346, correspondente à universidade vinculada.

\section{RESULTADOS}

\section{Caracterização das participantes}

As participantes desta pesquisa foram 10 mães de filhos com histórico de alteração de frênulo lingual. Destas, seis residiam em Santa Maria, com idades entre 18 e 41 anos. Em relação ao estado civil, cinco eram casadas e cinco eram solteiras. O número de filhos foi variável: quatro entrevistadas relataram possuir um filho, três possuíam dois filhos, duas com três filhos e uma com oito filhos. Sobre o índice de escolaridade materna, quatro participantes informaram ter Ensino Fundamental Incompleto, duas Ensino Médio Incompleto, três Ensino Médio Completo e uma Ensino Médio Completo e curso Técnico. No que se refere ao emprego, cinco entrevistadas estão empregadas, sendo uma em serviços de limpeza, outra em conf eitaria e as demais não informaram a ocupação.

Em relação às perguntas sobre a gestação e o parto, nove participantes realizaram o pré-natal com início no primeiro trimestre e uma não realizou por dificuldade de aceitação da gestação. Quando questionadas sobre intercorrências na gestação, sete afirmaram a ocorrência, sendo que três foram diagnosticadas com HAS, uma com sífilis e pré-eclâmpsia, uma com infecção urinária e HAS, uma com diabetes ges tacional e uma com crescimento intrauterino restrito. Das participantes que apresentaram alterações, somente duas fizeram uso de medicação durante a gestação.

Quanto ao parto, três necessitaram induzir o trabalho de parto, duas necessitaram de cesariana devido ao diagnóstico de HAS e uma por sofrimento fetal. Após o parto, uma apresentou hemorragia, uma necessitou de histerectomia parcial de emergência e em uma foi constatado múltiplos nódulos uterinos. Duas negaram intercorrências durante o parto. Todas relataram que seus filhos realizaram os exames de triagem neonatal no hospital.

No que diz respeito à presença de alteração de frênulo lingual na família, seis mães informaram possuir histórico: um pai e uma tia materna, um primo paterno, uma tia paterna, um tio materno e um irmão. $O$ diagnóstico de alteração de frênulo lingual foi realizado no hospital, durante o período de internação após o nascimento do bebê, sendo que das 10 crianças, seis apresentaram escore de 7 no protocolo de Triagem de Avaliação do Frênulo Lingual, indicando a necessidade da realização da frenotomia e três apresentaram escore de 5/6, apontando a necessidade de reavaliação após 30 dias. A idade das crianças no momento da entrevista variou de quatro meses a um ano e um mês.

A partir da análise temática foram construídas duas categorias, descritas a seguir. 


\section{Implicações da alteração de Frênulo Lingual no Aleitamento Materno Exclusivo}

Algumas entrevistadas relataram dificuldades no aleitamento materno nos primeiros dias, ocasionadas pela dor durante a mamada, fissuras mamilares e necessidade uso de bico intermediário de silicone.

"Foi bem ruim essa parte da pega, ele não conseguia pegar, tive que usar aquele bico de silicone para ele poder mamar. O bico (mamilo) foi melhorando, aí ele foi conseguindo pegar, fui me recuperando para não machucar muito meu peito, cicatrizou tudo. Eu fui retornando nas consultas no hospital e ela (pediatra) foi dizendo para começar a tirar o bico de silicone, oferecer o peito sem o bico e eu fui indo, não desisti”. (P2)

"Não consegui amamentar, ela não pegava. $(P 4)$

"Tive dificuldade para amamentar nos primeiros dias, machucou bastante porque ele não fazia a pega correta, tive que usar o bico de silicone. Usei por uma semana, depois ele pegou certinho". (P8)

As participantes informaram sobre o tempo prolongado da mamada, baixo ganho de peso e indicação para uso de fórmulas lácteas infantis.

"Eu amamentei um mês e meio, porque o meu peito não tem bico (mamilo) e ele tem a língua meio presa. Era difícil a sucção para ele, demorava bastante. Ele não estava ganhando peso, aí levei ele no médico e começaram a dar a fórmula. Ele começou a largar do peito". (P1)

"No primeiro mês insisti muito, mas ela foi largando, foi bem difícil, eu não produzia, pois ela não conseguia sugar por muito tempo. Tive fissuras nos primeiros dias, mas tive bastante orientação. Vim para casa dando no copinho. Ela era muito pequena, parecia não ter muita força, cansava e chorava. Fiz de tudo, mas não deu". (P5)

"Foi bem difícil, tanto que quando a neném estava com 6 dias voltamos para o pronto atendimento pediátrico porque ela teve um episódio de hipoglicemia. Quando saímos do hospital novamente tivemos a indicação da fórmula, ela mamou no peito por quase dois meses e depois não quis mais pegar". ( $P 9)$

Em contrapartida, algumas participantes negaram intercorrências no aleitamento materno como dor, fissuras mamilares ou outras dificuldades, e relataram conseguir manter o aleitamento materno exclusivo apesar das alterações de frênulo lingual.

"Foi super tranquilo, não machucou e não tive dificuldades". (P6)

"Ele só mama no peito, mama bem, tenho bastante leite. Amamentei todos, o G. ainda mamava quando o F. nasceu. Depois que ele nasceu, deixei o peito só pra ele. Eu gosto de dar de mamar, eu adoro ver eles se alimentando em mim. Não, tenho dificuldade para amamentar. (P7)

"Bem tranquilo, o que eu acredito que seja normal, porque como os dois tiveram a língua presa. Estou amamentando, em livre demanda, só não mama a hora que não estou. Tentei dar mamadeira para ver se ele mamava o tempo que eu não estou, mas não quis". (P10)

\section{Orientações para reavaliação do Frênulo Lingual}

Quando questionadas sobre a necessidade de retorno ao hospital para reavaliação de frênulo lingual, as entrevistadas relataram que retornaram às consultas para avaliação com cirurgião pediátrico, a fim de verificar a necessidade da realização de frenotomia. Em alguns casos, foi observado não haver a necessidade do procedimento. 
"Eu voltei lá, o doutor fez uma avaliação e me disse como ele não falava ainda, porque tinha 6 meses, não tinha como saber se iria atrapalhar ou não. Como ele estava ganhando peso, não estava atrapalhando a amamentação, por enquanto ele iria optar por não fazer a cirurgia". (P2)

"As médicas olharam ele e falaram que não ia ter a língua presa, que não ia ser preciso a cirurgia. Agora ele está com 1 aninho e eu não reparo nada, ele coloca a linguinha para fora, bem normal. Eu não fui mais aí no hospital (hospital onde ocorreu o nascimento), não quiseram marcar consulta mais para ele retornar, a médica falou que não ia mais precisar, que era para mim cuidar em casa". (P3)

"Nas primeiras consultas perguntei para pediatra e ela disse que não era o caso, já que não atrapalhava na amamentação. Mas, com o tempo foi formando esse buraquinho (local onde o frênulo se inseria)". (P8)

Algumas mães informaram que houve a necessidade de realizar a frenotomia devido à dificuldade para pega correta e, consequente, sucção na hora da mamada.

"Eu retornei para fazer o cortezinho com um mês e pouco. Era para ela ter retornado com 6 meses (reavaliação), só que aí estava essa confusão por causa da pandemia. Não notei diferença no aleitamento após a frenotomia". (P6)

"Fez a cirurgia (frenotomia). Sinceramente não vi diferença nenhuma, mas olhando a linguinha dele estava mesmo bem pega". (P7)

"Ela fez a frenotomia com 15 dias de vida. Depois ela melhorou a pega, acredito que se eu houvesse insistindo mais ela ainda estaria no peito, com o medo de que a glicose dela baixasse e com a falta de informações sobre amamentação e pega correta, eu acabava dando a fórmula". (P9)

Destaca-se que algumas participantes informaram possuir consulta agendada, mas em decorrência da pandemia da COVID-19 houve o cancelamento ou adiamento da consulta.

"O hospital havia encaminhado, mas logo veio a pandemia e foi tudo adiado, estou aguardando ser chamada. Temos medo de que atrapalhe a fala dela". (P5)

"Até o A. tinha retorno com a fonoaudióloga em abril para avaliar, para depois fazer a cirurgia. Mas, como começou a pandemia a gente não quis ir. Até agora nem sei o que a gente faz, se a gente espera ficar maior ou enquanto é menor". (P10)

\section{DISCUSSÃO}

O aleitamento materno é preconizado de forma exclusiva até o $6^{\circ}$ mês de vida, podendo estender-se até os dois anos ou mais, devido aos benefícios imunológicos, nutricionais e emocionais para o bebê e para a mulher (MINISTÉRIO DA SAÚDE, 2015b; ALMEIDA JM, et al., 2015). Embora existam recomendações para esta prática, ainda é observado uma significativa prevalência de desmame precoce (BARBOSA GEF, et al., 2017).

Para que o aleitamento materno se estabeleça fazem-se necessários alguns fatores como o desejo materno e o aprendizado durante o processo de amamentação, tanto da mãe quanto do bebê, o qual pode ser vivenciado de forma positiva ou negativa para a continuidade do aleitamento materno (BROWN A, 2017).

Durante o período de amamentação, muitas dificuldades podem surgir, principalmente, em relação à dor durante a amamentação, produção láctea, fatores psicossociais, situação nutricional e de satisfação do bebê, dificuldades de posicionamento e pega do bebê na mama, fazendo com que possam se constituir em importantes fatores de risco para o desmame precoce. Somado a isso, a falta de apoio de profissionais de saúde para a manutenção do aleitamento, como indicação de bicos artificiais e uso de fórmula logo após o 
nascimento, contribuem para uma diminuição na adesão ao aleitamento materno (CARREIRO JA, et al., 2018; BROWN A, 2017).

$\mathrm{Na}$ literatura, encontra-se uma variabilidade na prevalência de anquiloglossia. Estudo realizado para avaliar o frênulo da língua em bebês recém-nascidos a termo e verificar sua associação com o aleitamento materno, demonstra uma prevalência de $0,8 \%$ de um total de 139 bebês avaliados (FUJINAGA IC et al., 2017).

Já outro estudo que aplicou um protocolo de avaliação de frênulo lingual para constatar a existência ou não de fatores que influenciam as funções de sucção e deglutição em bebês nascidos a termo, relatou a prevalência de anquiloglossia referente a $22,5 \%$, resultando em restrição do movimento da língua na sucção devido às características anatômicas (MARTINELLI RLC, et al., 2013).

Assim, evidências científicas demonstram que bebês que apresentam anquiloglossia podem possuir dificuldades em realizar uma adequada extração de leite, causando dores durante a mamada, baixo ganho de peso e tempo de mamada prolongado. Além disso, essa anomalia congênita pode ocasionar uma baixa produção láctea pela puérpera já que a pega inadequada pode acarretar menor estimulação da mama (POMINI MC, et al., 2018; MARTINELLI RLC, et al., 2016).

Embora a anquiloglossia seja um fator influente na prática do aleitamento materno, outras variáveis também devem ser consideradas nos casos em que ocorrem o desmame, seja ele precoce ou não. A amamentação abrange fatores naturais e culturais, sendo um processo não somente biológico, mas socialmente construído, impregnado de ideologias (FUJINAGA IC, et al., 2017).

Estudo desenvolvido para analisar a associação entre o tipo de aleitamento materno e as dificuldades relacionadas à essa prática, evidenciou que mulheres com experiência prévia, maior escolaridade materna, percepção materna da quantidade de leite e percepção materna das mamas antes e após as mamadas apresentaram maiores chances de manter o aleitamento materno (CARREIRO JA, et al., 2018).

Somado a isso, orientações sobre o aleitamento materno durante o pré-natal, parto e puerpério contribuem para a superação de dificuldades, bem como auxiliam para um melhor manejo mesmo em bebês que possuem anquiloglossia (CARREIRO JA, et al., 2018; FUJINAGA IC, et al., 2017; ALMEIDA JM, et al., 2015).

As orientações sobre amamentação, desde o pré-natal, devem prezar pela compreensão e escuta do conhecimento da mulher, fornecendo informações que permitam sua reflexão e decisão (BATISTA MR, et al., 2017). Também, cabe a equipe de saúde a orientação sobre a importância da avaliação do frênulo lingual, tendo em vista que a descoberta de alterações possibilita desenvolver estratégias que auxiliem no processo de amamentação ou, se necessário, a realização do tratamento preco ce (PENHA S, et al., 2019).

O tratamento da anquiloglossia é cirúrgico (frenotomia), sendo indicado quando o frênulo lingual restringe ou dificulta a movimentação da língua. A indicação para frenotomia deve ser realizada o mais precocemente possível, destacando a atuação de profissionais de saúde como médicos pediatras, enfermeiros, fonoaudiólogos e odontopediatras, desde a identificação e visualização de fatores indicativos, até a realização de intervenção adequada e efetiva. Entretanto, ainda há divergências entre profissionais a respeito do risco do procedimento e de benefícios como melhor manejo das dificuldades durante a amamentação (PROCOPIO IMS, et al., 2017; JUNQUEIRA MA, et al., 2014).

Pesquisa que apresentou diversas técnicas para correção de anquiloglossia (JUNQUEIRA MA, et al., 2014), aponta que após realizado o diagnóstico de alteração de frênulo, ainda existem divergências sobre o manejo destes pacientes, visto que não há um consenso sobre indicação, tempo para intervenção e técnica cirúrgica apropriada. A frenotomia é a técnica mais indicada para lactentes, já que se constitui em um procedimento conservador, simples e de fácil realização, sendo de competência médica ou odontóloga a sua realização.

Frente a isso, verifica-se a necessidade de um maior desenvolvimento de estudos sobre a temática com o objetivo de padronizar as técnicas utilizadas para diagnóstico e tratamento de forma precoce, para auxiliar a díade mãe-bebê que enfrenta dificuldade na prática do aleitamento materno exclusivo. 


\section{CONCLUSÃO}

Dos 10 bebês que apresentaram anquiloglossia, sete não necessitaram realizar o procedimento de frenotomia quando reavaliados no retorno da consulta médica. Entretanto, observou-se que as dificuldades durante a amamentação podem estar relacionadas à interferência do frênulo lingual durante o processo de sucção do bebê. Tais dificuldades resultaram em baixo ganho de peso em alguns bebês, fadiga e tempo de mamada prolongado, além de fissuras mamilares apresentadas pelas lactantes. Evidenciou-se, também, a necessidade de maior capacitação da equipe multiprofissional de saúde para fornecer informações integradas, de forma a auxiliar na superação das dificuldades, diminuição do desmame precoce, além de evitar alterações futuras de motricidade orofacial e fala da criança.

\section{REFERÊNCIAS}

1. ALMEIDA JM, et al. Apoio ao aleitamento materno pelos profissionais de saúde: revisão integrativa de literatura. Revista Paulista de Pediatria, 2015; 33(3):355-362.

2. ARAUJO MCM, et al. Avaliação do frênulo lingual em recém-nascidos com dois protocolos e sua relação com o aleitamento materno. Jornal de Pediatria, 2020;96(3): 379-385.

3. BARBOSA CEF, et al. Dificuldades iniciais com a técnica da amamentação e fatores associados a problemas com a mama em puérperas. Revista Paulista de Pediatria, 2017;35(3):265-272.

4. BATISTA MR, et al. Orientações de profissionais da saúde sobre aleitamento materno: o olhar das puérperas. Joumal of Nursing and Health, 2017;7(1): 25-37.

5. BRAUN V, CLARKE V. Using thematic analysis in psychology. Qualitative Research in Psychology, 2006; 3(2): 77101.

6. BROWN A. Breastfeeding as a public health responsibility: a review of the evidence. Journal of Human Nutrition and Dietetics, 2017;30(6).

7. CARREIRO JA, et al. Dificuldades relacionadas ao aleitamento materno: análise de um serviço e specializado em amamentação. Acta Paulista de Enfermagem, 2018;31(4):430-8.

8. CRESWELL JW, CLARK VLP. Pesquisa de Métodos Mistos. 2ª ed. Porto Alegre:Penso, 2013.

9. FRAGA MRBA, et al. Anquiloglossia versus amamentação: qual a evidência de associação?. Revista CEFAC, 2020; 22(3): e12219.

10. FUJINAGA IC, et al. Frênulo lingual e aleitamento materno: estudo descritivo. Audiology - Communication Research (ACR), 2017;22: 1-7.

11. JUNQUEIRA MA, et al. Surgical techniques for the treatment of ankyloglossia in children: a case series. Journal of Applied Oral Science, 2014;22(3): 241-8.

12. MARTINELLI RLC, et al. Validade e confiabilidade da triagem: "teste da linguinha". Revista CEFAC, 2016; 18(6): 1323-1331.

13. MARTINELLI RLC, et al. Protocolo de avaliação do frênulo lingual para bebês: relação entre aspectos anatômicos e funcionais. Revista CEFAC, 2013; 15(3): 599-610.

14. MINISTÉRIO DA SAÚDE. Conselho Nacional de Saúde. Resolução n. 466, de 12 de dezembro de 2012. Aprova diretrizes e normas regulamentadoras de pesquisas envolvendo seres humanos. 2012. Disponível em: https://bvsms.saude.gov.br/bvs/saudelegis/cns/2013/res0466_12_12_2012.html Acesso em: 18 de novembro de 2021.

15. MINISTÉRIO DA SAÚDE. Conselho Nacional de Saúde. Resolução no 510, de 7 de abril de 2016. Dispõe sobre as normas aplicáveis a pesquisas em Ciências Humanas e Sociais. 2016. Disponível em: http://conselho.saude.gov.br/resolucoes/2016/Reso510.pdf Acesso em: 18 de novembro de 2021.

16. MINISTÉRIO DA SAÚDE. Conselho Nacional de Saúde. Resolução no 580 , de 22 de março de 2018. Dispõe sobre as especificidades éticas das pesquisas de interesse estratégico para o Sistema Único de Saúde (SUS) serão contempladas em Resolução específica, e dá outras providências. 2018. Disponível em: https://conselho.saude.gov.br/resolucoes/2018/Res0580.pdf Acesso em:18 de novembro de 2021.

17. MINISTÉRIO DA SAÚDE. Secretaria de Atenção à Saúde. Departamento de Atenção Básica. Caderno de Atenção Básica $n^{\circ} 23:$ Saúde da Criança - aleitamento materno e alimentação complementar. 2015a. Disponível em: Acesso em: https://bvsms.saude.gov.br/bvs/publicacoes/saude_crianca_aleitamento_materno_cab23.pdf Acesso em: 18 de novembro de 2021.

18. MINISTÉRIO DA SAÚDE. Portaria no 1.130 , de 5 de agosto de 2015. Institui a Política Nacional de Atenção Integral à Saúde da Criança (PNAISC) no âmbito do Sistema Único de Saúde (SUS). 2015b. Acesso em: 18 de novembro de 2021.

19. NOGUEIRA JS, et al. Frenotomia: da avaliação à intervenção cirúrgica. Revista CEFAC, 2021;23(3): e10420.

20. O'SHEA JE, et al. Frenotomy for tongue-tie in newborn infants. Cochrane Database of Systematic Reviews, 2017;3: D011065.

21. PADOIN SMM, et al. Entrevista telefônica como técnica de coleta de dados. Cadernos de Ciência e Saúde, 2013; 24(3): 95-100.

22. PENHA S, et al. Teste da linguinha: as gestantes sabem do que se trata?. Revista Eletrônica Acervo Saúde, 2019; 11(13): e957.

23. POMINI MC, et al. Conhecimento de gestantes sobre o teste da linguinha em neonatos. Revista de Odontologia da UNESP, 2018;47(6): 341-47.

24. PROCOPIO IMS, et al. Frenotomia lingual em lactentes. Revista da Faculdade de Odontologia - UPF, 2017; 22(1): 114-119. 